\title{
Mejora del manejo del riego por surcos en el Bajo Guadalquivir.
}

\author{
Salvatierra Bellido, B. ${ }^{1}$, Jarén Morilla, C. ${ }^{1}$, López Rodríguez, M. ${ }^{1}$
}

Instituto de Investigación y Formación Agraria de Andalucía, Rancho de la Merced (IFAPA, sede Chipiona-Cádiz; benito.salvatierra@juntadeandalucia.es, cristina.jaren@juntadeandalucia.es, manuel.lopez.rodriguez@juntadeandalucia.es

Resumen: El riego por surcos es todavía un método utilizado en muchas zonas regables por las características intrínsecas a las mismas, bien por la ausencia de costes energéticos o bien por no haber acometido proyectos de presurización de redes de distribución de agua. El problema principal del manejo del riego en general es conseguir eficiencia y a la vez reducir la constante lixiviación de nitratos. El riego por gravedad representa en Andalucía un 15\% de la superficie de regadío y concentrada una gran parte en el Bajo Guadalquivir en zonas no modernizadas. En el año 2011 se empezo a estudiar la eficiencia del riego por surcos en el Bajo Guadalquivir desde el Instituto de Andaluz de Investigación y Formación Agraria, Pesquera, Alimentaria y de Producción Ecológica (IFAPA) dónde desde Centro de Chipiona se hizo la primera evaluación de riego por surcos sobre un cultivo de algodón. Con estos datos de partida obtenidos, el objetivo marcado es conseguir una mejor eficiencia de aplicación del agua en el riego por surcos, junto con la mayor eficiencia en la aplicación del abonado nitrogenado mejorando la rentabilidad de las explotaciónes. Para ello, se implementa en un ensayo la tecnología del riego por surcos por pulsos para reducir el consumo de agua y disminuir las tasas de lixiviación de nitratos a través de la fertirrigación en esta modalidad de riego. Para ello, se ha contado con una parcela colaboradora con características representativa de la zona, dónde se compara en el cultivo del algodón con dos manejos diferentes. Por un lado, el riego convencional por surcos con abonado convencional a voleo frente al riego por pulsos con fertirrigación automatizada. Cuando se riega por pulsos en el riego por surcos se aplica un caudal por cada surco mayor, además de que cada pulso de riego se realiza el avance sobre suelo saturado y por consiguiente con un mayor grado de impermeabilidad en la zona ya regada. El sistema permite la inyección del abonado en función a la fase de riego más idónea. La aplicación de esta tecnología ha supuesto: Una mayor eficiencia de riego con un ahorro de agua del $25 \%$ para las condiciones del ensayo. Esta situación de ahorro de agua también permitirá mayor frecuencia de riego, redundando sobre mejores rendimientos de los cultivos. Igualmente en el manejo del abonado se consigue un aumento en la eficiencia del abonado nitrogenado con un ahorro del 34\% aproximadamente en las condiciones del ensayo. Finalmente, los resultados obtenidos mejorarán y la aplicación de esta técnica mejorarán la productividad y sostenibilidad de los cultivos con esta modalidad de riego por surcos.

Palabras clave: Riego; surcos; pulsos; fertirrigación. 


\title{
XXVIII Congreso Nacional de Riegos CARTAGENA 2021
}

\section{Improvement of furrow irrigation management in the Lower Guadalquivir.}

\author{
Salvatierra Bellido, B. ${ }^{1}$, Jarén Morilla, C. ${ }^{1}$, López Rodríguez, M. ${ }^{1}$
}

Instituto de Investigación y Formación Agraria de Andalucía, Rancho de la Merced (IFAPA, sede Chipiona-Cádiz; benito.salvatierra@juntadeandalucia.es, cristina.jaren@juntadeandalucia.es, manuel.lopez.rodriguez@juntadeandalucia.es

\begin{abstract}
Furrow irrigation is still a method used in many irrigable areas due to their intrinsic characteristics, either due to the absence of energy costs or because they have not undertaken pressurization projects for water distribution networks. The main problem of irrigation management in general is to achieve efficiency while reducing the constant leaching of nitrates. Irrigation by gravity represents $15 \%$ of the irrigated area in Andalusia and a large part is concentrated in the Lower Guadalquivir in non-modernized areas. In 2011, the efficiency of furrow irrigation in the Lower Guadalquivir began to be studied by the Instituto de Andaluz de Investigación y Formación Agraria, Pesquera, Alimentaria y de Producción Ecológica (IFAPA), where the Center of Chipiona made the first evaluation of furrow irrigation on a cotton crop. With these starting data obtained, the objective is to achieve a better efficiency in the application of water in furrow irrigation, together with greater efficiency in the application of nitrogen fertilizer, improving the profitability of the farms. For this, the technology of surge furrow irrigation is implemented in a trial to reduce water consumption and reduce nitrate leaching rates through fertigation in this irrigation modality. For this, a collaborating plot with representative characteristics of the area has been counted on, where it is compared in the cultivation of cotton with two different operations. On the one hand, conventional furrow irrigation with conventional broadcast fertilizer versus surge irrigation with automated fertigation. When irrigating by surge irrigation, a flow is applied for each larger furrow, in addition to each irrigation pulse the advance is carried out on saturated soil and therefore with a greater degree of impermeability in the already irrigated area. The system allows the injection of the fertilizer according to the most suitable irrigation phase. The application of this technology has meant: Greater irrigation efficiency with a water saving of $25 \%$ for the test conditions. This situation of saving water will also allow a greater frequency of irrigation, resulting in better crop yields. Likewise, in the handling of the subscriber, an increase in the efficiency of the nitrogen subscriber is achieved with a saving of approximately $34 \%$ under the test conditions. Finally, the results obtained will improve and the application of this technique will improve the productivity and sustainability of the crops with this modality of furrow irrigation.
\end{abstract}

Keywords: Irrigation; furrow; surge; fertigation. 


\section{Congreso Nacional de Riegos CARTAGENA 2021}

\section{Introducción}

El riego por surcos es una variante del sistema de riego por gravedad que predomina en muchas zonas de España y que actualmente es uno de los más sostenible energéticamente. La superficie de riego por gravedad en España aún tiene presencia en el regadío español con unas 908.075 ha de las cuales Andalucía representa el $18,7 \%$ con 169.875 ha [1].

La mejora de la eficiencia en el uso del agua en el riego por surcos (Walter, 1989) está muy ligada al diseño previo y definitivo de las condiciones de la parcela: tipo de suelo, pendiente y longitud del recorrido del agua. Pero existen otras variables como caudal de riego y la humedad inicial antes de un riego, que pertenecen al manejo del riego en cada momento y son de carácter circunstancial. La variación de estas últimas para la mejora de la eficiencia de riego requieren de conocimientos técnicos muy cualificados.

Desde el Instituto Andaluz de Investigación y Formación Agraria, Pesquera, Alimentaria y de la Producción Ecológica (IFAPA) y desde el Centro Rancho de la Merced, se están desarrollando líneas de trabajo para mejorar la eficiencia del riego, del abonado y la fertirrigación en cultivos herbáceos de regadío al aire libre. La mayoría de la superficie de riego por surcos está incluida en estos sistemas de cultivo y por tanto requiere de un estudio específico para hacer más sostenible este sistema de riego . Desde los proyectos de transferencia de tecnología en el regadío y de optimización del abonado nitrogenado, se ha concluido con la puesta a punto un sistema de riego innovador, que hace más eficiente el riego por gravedad, a la vez que permite la aplicación de abono con mínimas tasas de percolación profunda.

El riego por gravedad es el único método que prescinde del consumo energético y es por tanto el más económico, pero a su vez es uno de los sistemas menos tecnificados y dónde la baja eficiencia de aplicación del agua merma mucho las cosechas por la distribución irregular del agua y la lixiviación de los nitratos. Por tanto, investigar sobre este sistema de riego mejorará la eficiencia de aplicación [2] consiguiendo disponer de producciones similares a las de un riego a presión.

Por todo y desde el IFAPA se ha analizado, en condiciones experimentales sobre el cultivo del algodón, la introducción de mejoras en el riego por surcos, dónde se ha utilizado el riego por surcos en la modalidad de aplicación por pulsos de riego [3] y con inyección del abono a través de este sistema de riego.

\section{Objetivo}

El fin último de este trabajo es conseguir un manejo más sostenible del riego por surcos. Dónde los objetivos específicos son los siguientes:

1. Conseguir mayor eficiencia en el riego por sucos;

2. Conseguir mayor eficiencia en el abonado en el cultivo del algodón y todos los cultivos regados con este sistema en general;

3. Hacer más sostenible cualquier cultivo con este sistema, tanto desde el punto de vista ambiental y como de rentabilidad.

\section{Materiales y métodos}

\subsection{Materiales}

Para el estudio se contó con una parcela en la zona regable del Bajo Guadalquivir, en la Comunidad de Regante de Las Marismas en la provincia de Sevilla. Para ello se utilizó una parcela de 4 ha de 


\section{Congreso Nacional de Riegos CARTAGENA 2021}

superficie la cual se dividió en dos subpardes de 2 ha cada una y con propiedades similares. Dicha parcela fue escogida como representativa y proporcionada a través de un convenio del IFAPA con el Colectivo de Algodoneras Del Sur De Andalucía S.L. (COALSA). El suelo es muy homogéno de textura arcillosa y con una conductividad eléctrica en extracto $1 / 5$ que oscilaba desde los $0,244 \mathrm{dS} / \mathrm{m}$ en los primeros 30 centímetros a los $0,859 \mathrm{dS} / \mathrm{m}$ en los siguientes 30 centímetros, y partiendo de un nivel de nitratos en el suelo de $132 \mathrm{mg} / \mathrm{kg}$ a los $40 \mathrm{mg} / \mathrm{kg}$ respectivamente. Toda la parcela está drenada con drenes subterráneos a una profundidad de $0,8 \mathrm{~m}$ y una equidistancia de $5 \mathrm{~m}$. La dirección de los drenes es la misma a las líneas de cultivo (de Sur a Norte).

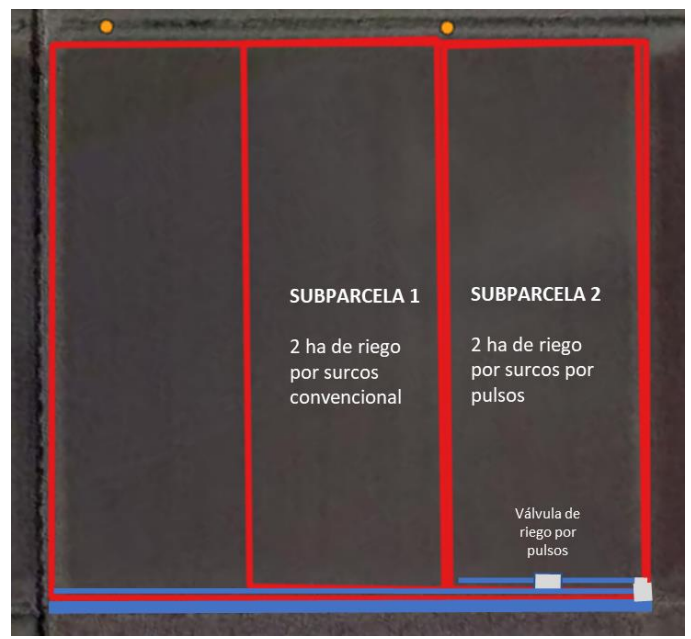

Figura 1. Croquis de las dos parcelas experimentales de riego por sucos. De derecha a izquierda la subparcela de manejo más sostenible y la subparcela de riego convencional. Los puntos en amarillos son los puntos colectores de drenaje hacia un canal colector de drenaje.

Sobre las dos parcelas se cultivó algodón con los objetivos de cubrir las necesidades de riego a través de riego por surcos en ambas subparcelas.

El cultivo se siembra el 2 de abril de 2021 y se recolecta el 21 de septiembre del mismo año. Ambas subparcelas tienen el mismo manejo excepto las prácticas de riego y abonado, por las que son objeto de este estudio. Por tanto, se distinguen dos manejos distintos:

- Subparcela 1. En dicha parcela se lleva a cabo el manejo convencional de riego y abonado que se hace tradicionalmente en la zona y que se describe más adelante

- Subparcela 2. En esta por el contrario se aplica una técnica innovadora de riego por surcos: riego por pulsos. Y la práctica del abonado se hace a través de la fertirrigación

Para la subparcela 2 se ha utilizado una válvula de riego para riego por surcos que proporciona riego por pulsos. Es decir, este elemento en forma de pieza en " $\mathrm{T}$ " (figura 2) se encarga de aplicar agua sobre los surcos de forma intermitente sobre dos tuberías de plastocanal situadas a ambos lados de la válvula. La función de la válvula es conmutar cada cierto tiempo, el agua recogida por la acequia de distribución, hacia un lado u otro. La frecuencia y tiempo de cambio está gobernada por unos algoritmos predefinidas en el autómata de la válvula. Aunque en cualquier momento puede ser modificado por el usuario.

Todo el sistema es autónomo y automático, ya que se alimenta energéticamente a través de una placa fotovoltaica y una batería que asegura el funcionamiento en ausencia de luz.

El objetivo del sistema es regar de mara óptima para evitar la mayor cantidad de infiltración en cabecera y hacer el riego lo más uniforme posible. Esto se consigue saturando de manera rápida los 


\section{Congreso Nacional de Riegos CARTAGENA 2021}

tramos iniciales en los primeros avances del agua y caudales el doble a un riego por surco convencional, de esta manera conseguimos rápidamente un suelo más impermeable asegurando avances más rápidos en los siguientes pulsos y de manera progresiva.

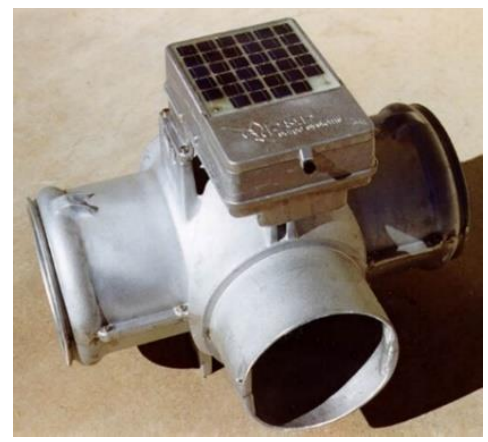

Figura 2. Imagen de la válvula para el riego por pulsos en riego por surcos.

Simultáneamente el dispositivo puede llevar asociado un equipo de fertirrigación (figura 3), a la vez que puede ser programado para ello. Dicha programación optimiza el reparto de abono en una fase posterior a la fase de avance del agua en el surco. A dicha fase se le denomina fase de remojo e inyecta el abono con pulsos de riego con una mayor frecuencia.

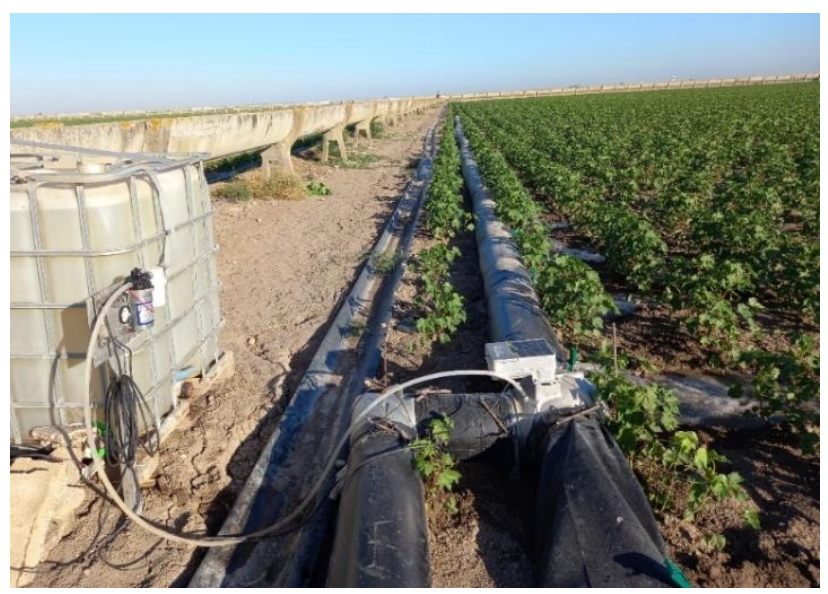

Figura 3. Imagen del sistema en funcionamiento con el sistema de fertirrigación incorporado.

En la figura anterior se muestra la instalación del ensayo de algodón realizado en el año 2021 sobre la parcela experimental y con el cultivo del algodón. La válvula de riego por surcos soporta a cada lado de ella dos tuberías de plastocanal de $400 \mathrm{~mm}$ de diámetro y de una longitud cada una de 22,5 m. Sobre estas tuberías flexibles se han instalados compuertas para cada suco con una equidistancia de 1,5 $\mathrm{m}$ regando calles alternas del cultivo.

\subsection{Métodos}

Una vez dispuesto el sistema de riego por pulsos en la subparcela 2 de riego por surcos y el cultivo ya implantado en la fase de nascencia se propuso la siguiente metodología.

Al tratarse de dos parcelas experimentales únicas, con diferentes variables de manejos y dado el tamaño de estas (subparcela 1 y 2), fue imposible disponer de un diseño experimental alternativo que 


\section{Congreso Nacional de Riegos CARTAGENA 2021}

permitiese un tratamiento estadístico posterior. Por tal motivo se llevó a cabo un seguimiento del desarrollo del cultivo a través de imágenes satélites. De esta manera se pudieron analizar los resultados con mayor precisión.

Una vez definido el objetivo de manejo de cada parcela, se expone a continuación los tipos de operaciones realizadas sobre cada una.

- Subparcela 1. El manejo del riego y el abonado corrió a cargo del agricultor y reproduciendo el sistema de cultivo representativo de la zona regable. En este caso el riego se realizaba con una sola tubería de plastocanal similar al sistema de riego por pulsos, pero con taladros en la tubería a la altura de cada surco (alternos a las líneas de cultivo igualmente). El manejo de abonado consistió en un abonado de fondo 5 días antes de la siembra y un abonado de cobertera justo antes del segundo riego. Las dosis de todas las aplicaciones se muestran en el apartado de resultados.

- Subparcela 2. En este caso el riego fue por pulsos y adaptado a la frecuencia impuesta por la petición de agua solicitada a la comunidad de regantes por parte del agricultor. Y el abonado en este caso, y por asegurar el inicio del cultivo no fuera a llover y no se pudiera fertirrigar se aplicó un abonado de fondo con menor dosis que el agricultor. Y posteriormente se fertirrigó en función a las curvas de absorción de nutrientes del cultivo y adaptada a la frecuencia de los riegos.

\section{Resultados y discusión}

Después de todo el cultivo y sobre una campaña de riego completa, los resultados han arrojado una gran cantidad de información que merece ser analizada por partes como consecuencia de la comparación de un manejo tradicional de los factores riego y abonado frente a un manejo optimizado.

\subsection{Riego}

Los parámetros de riego recogido de ambas subparcelas se recogen a continuación para definir las diferencias entre ambos manejos desde el punto de vista sólo de la aplicación del agua.

Se ha evaluado el caudal instantáneo de riego de cada chorro de agua obteniendo valores similares en todos los riegos y con los siguientes valores medios.

Tabla 1. Caudal instantáneo de cada chorro del riego por surcos.

\begin{tabular}{lcc}
\hline & $\begin{array}{c}\text { Subparcela 1 (manejo } \\
\text { convencional) }\end{array}$ & $\begin{array}{c}\text { Subparcela 2 (manejo } \\
\text { sostenible por pulsos) }\end{array}$ \\
\hline Caudal por chorro $(1 / \mathrm{s})$ & 0,8 & 1,5 \\
\hline
\end{tabular}

Por tanto, el caudal instantáneo en ambas parcelas fue similar y de $48 \mathrm{l} / \mathrm{s}$ ya que en el riego por surcos el caudal sólo iba hacia la mitad de la parcela de manera alterna e intermitente a cada zona.

El calendario de riego aplicado es el mismo en cada subparcela ya que se adpató a los turnos ya definidos por la comunidad de regantes con peticiones previas de los agricultores de la agrupación de parcelas dónde se situaba la parcela experimental. A continuación se sintetizan no sólo las fechas de los mismos sino además: el déficit de agua en el suelo [4] justo antes de cada riego y el porcentaje de reducción de tiempo de riego del manejo por pulsos frente al manejo convencional. Esta información se generó en el primer caso realizando un balance de agua en el suelo teniendo en cuenta la demanda evapotranspirativa del cultivo y el segundo caso comparando el tiempo en el que se completaba el 


\section{Congreso Nacional de Riegos CARTAGENA 2021}

riego en cada una de las subparcelas y por tanto mostrando la reducción del tiempo que se generaba regando por pulsos.

Tabla 2. Calendario de riegos, el Agua en el Suelo (DAS) justo antes del riego y el porcentaje de reducción de tiempo de riego en el riego por pulsos.

\begin{tabular}{cccc}
\hline RIEGO & Fecha & $\begin{array}{c}\text { DAS } \\
{[\mathrm{mm}]}\end{array}$ & $\begin{array}{c}\text { Porcentaje de reducción del tiempo de riego } \\
{[\%]}\end{array}$ \\
\hline $1^{*}$ & $13 / 04 / 2021$ & 25,7 & - \\
2 & $07 / 06 / 2021$ & 62,6 & 35,9 \\
3 & $29 / 06 / 2021$ & 85,5 & 17,1 \\
4 & $20 / 07 / 2021$ & 151,8 & 24,8 \\
5 & $04 / 08 / 2021$ & 108,8 & 22,8 \\
\hline
\end{tabular}

* Este riego fue convencional en ambas parcelas para no generar riesgos de nascencia del cultivo al agricultor

Exceptuando el primer riego, sobre cual no fue aplicada la técnica del riego por surcos, todos los riegos generaron una reducción de tiempo de riego. El riego 3, no consiguió una reducción de tiempo similar a los demás riegos, debido a que el sistema tuvo una falta de energía en la mitad del tiempo de riego y se apagó, a partir de ahí regamos manualmente. El motivo fue claramente porque la válvula fue guardada previamente al riego en un lugar cerrado no cargando la batería del riego anterior.

Por tanto y considerando esta irregularidad, se consiguió una reducción media de tiempo del $25 \%$ en el peor de los casos, y por tanto el dato merece un análisis de impacto en la mejora del riego por surcos. Dicha reducción de tiempo de riego es trasladable directamente a la reducción de volumen de agua.

En el siguiente gráfico se muestra la distribución de los riegos en función del Déficit de Agua en el Suelo (DAS) de partida y la reducción del tiempo de riego en el riego por pulso utilizado en la subparcela 2 frente al riego convencional aplicado en la subparcela 1

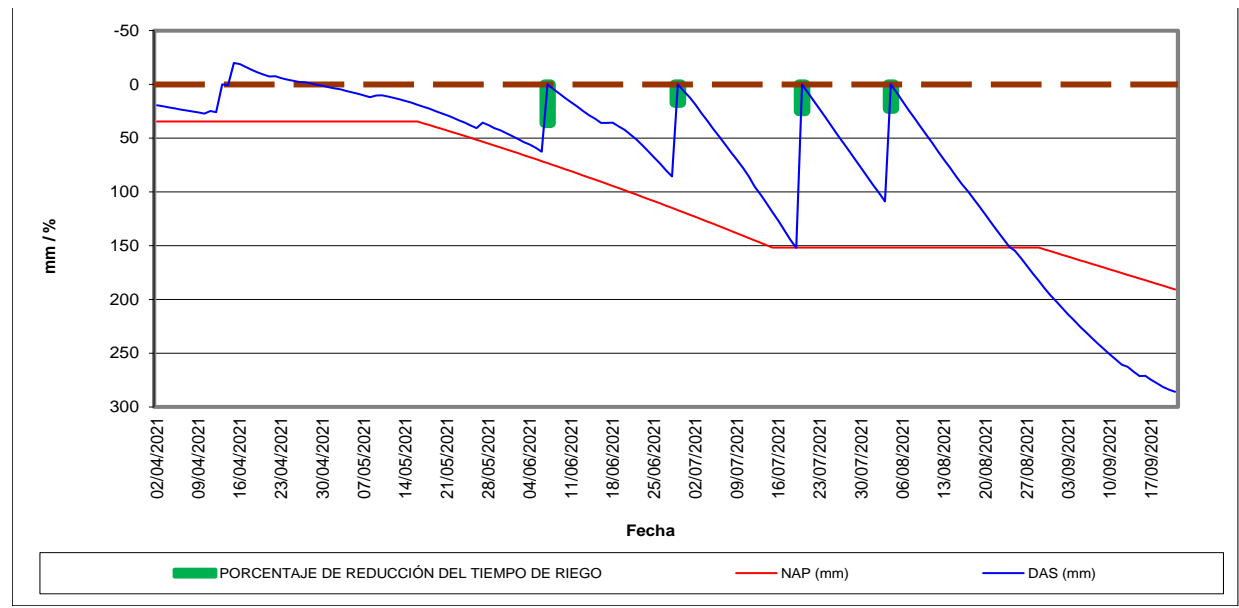

Figura 4. Gráfico del balance de agua en el suelo dónde se representa el DAS justo antes del riego el Nivel de Agotamiento Permisible (NAP) por el cultivo de algodón y el porcentaje de reducción de tiempo de riego en el riego por pulsos.

Habiendo medido los tiempos de riegos en cada subparcela y teniendo en cuenta el caudal instantáneo, los consumos de agua fueron los siguientes:

Tabla 1. Volúmenes de agua aplicados. 


\section{Congreso Nacional de Riegos CARTAGENA 2021

\begin{tabular}{ccc}
\hline & $\begin{array}{c}\text { Subparcela 1 (manejo } \\
\text { convencional) } \\
{\left[\mathrm{m}^{3} / \mathrm{ha}\right]}\end{array}$ & $\begin{array}{c}\text { Subparcela 2 (manejo } \\
\text { sostenible por pulsos) } \\
{\left[\mathrm{m}^{3 / \mathrm{ha}]}\right.}\end{array}$ \\
\hline $\begin{array}{c}\text { Volumen de agua } \\
\text { aplicado }\end{array}$ & 5.458 & 4.365 \\
\hline
\end{tabular}

* Estos volúmenes se han podido aplicar debido a la disponibilidad del agricultor en detrimento de riegos deficitarios en otros cultivos de agua en la explotación.

Un análisis a nivel de parcela del ahorro conseguido implicaría disponer directamente de un riego más sobre el cultivo. Pero además, esta circunstancia llevaría posiblemente a una mayor eficiencia de riego, al aumentar la frecuencia de los riegos. Esto produciría también una mayor producción del cultivo, incluso pudiendo llegar a mejorar la producción del algodón en riego por aspersión, puesto que la distribución del agua en el suelo podría ser más uniforme.

Y un análisis a mayor escala supondría que para un volumen ahorrado próximo a los $1.000 \mathrm{~m}^{3} / \mathrm{ha}$ y para una superficie estimada de riego por surcos en Andalucía de 50.000 ha se podría llegar a un ahorro de aproximadamente $50 \mathrm{hm}^{3}$

\subsection{Abonado}

Los parámetros de abonado recogidos en ambas subparcelas atiende a los criterios recogidos anteriormente.

- Sobre la subparcela 1 (parcela testigo) se distribuye el abono en una aplicación de fondo con dosis de abonado similar a la tradicional mas una sola aplicación de cobertera antes del segundo riego

- Sobre la subparcela 2 (parcela piloto) se distribuye en una aplicación de fondo reducida, con respecto a la parcela testigo mas cinco aplicaciones a través de fertirrigación y después de cada riego. Todas las aplicaciones se realizan según la curva de absorción de cada macronutriente del cultivo de algodón [5], dónde se aplicaba anticipadamente el equilibrio y dosis de abonado para el periodo posterior entre riego y riego.

El balance de distribución y dosis abono aplicado en unidades de fertilizantes se recoge en la siguiente tabla.

Tabla 3. Distribución de abonado en ambas subpacerlas.

\begin{tabular}{ccccccc}
\hline Tipo de aplicación & \multicolumn{3}{c}{ TESTIGO (subparcela 1) } & \multicolumn{2}{c}{ PILOTO (subparcela 2) } \\
\hline Macronutriente & $\mathbf{N}$ & $\mathbf{N}$ & $\mathbf{P}$ & $\mathbf{K}$ & $\mathbf{P}$ & $\mathbf{K}$ \\
Fondo & 32 & 24 & 60 & 15 & 96 & 32 \\
Cobertera/fertirrigación & 128 & 81 & 15 & 57 & - & - \\
$\quad$ TOTAL & $\mathbf{1 6 0}$ & $\mathbf{1 0 5}$ & $\mathbf{7 5}$ & $\mathbf{7 2}$ & $\mathbf{9 6}$ & $\mathbf{3 2}$ \\
\hline
\end{tabular}

Por tanto, en aplicación de nitrógeno se consigue un ahorro del 34,4 \% además del 21,6\% en fósforo.

El manejo sostenible mediante la fertirrigación también aportó las siguientes ventajas.

Un ahorro en la aplicación 


\section{Congreso Nacional de Riegos CARTAGENA 2021}

Una mayor eficiencia en la aplicación del abonado nitrogenado y por tanto una disminución de lixiviados. Todo ello teniendo en cuenta: el fraccionamiento de las aplicaciones, la aplicación sobre un suelo saturado y más impermeable y con la reducción de volumen agua de agua en el riego.

\subsection{Producción}

Para verificar la viabilidad del sistema de riego se ha evaluado tanto el cultivo como la estimación de la producción final. Para ello, no sólo se recolectaron de manera independiente ambas parcelas, sino que además, y para verificar el comportamiento de los suelos y del cultivo, se hizo un seguimiento con satélite. Para ello se recogieron valores de NVDI característicos de ambas parcelas dónde se incluye los valores máximos y mínimos de cada una.

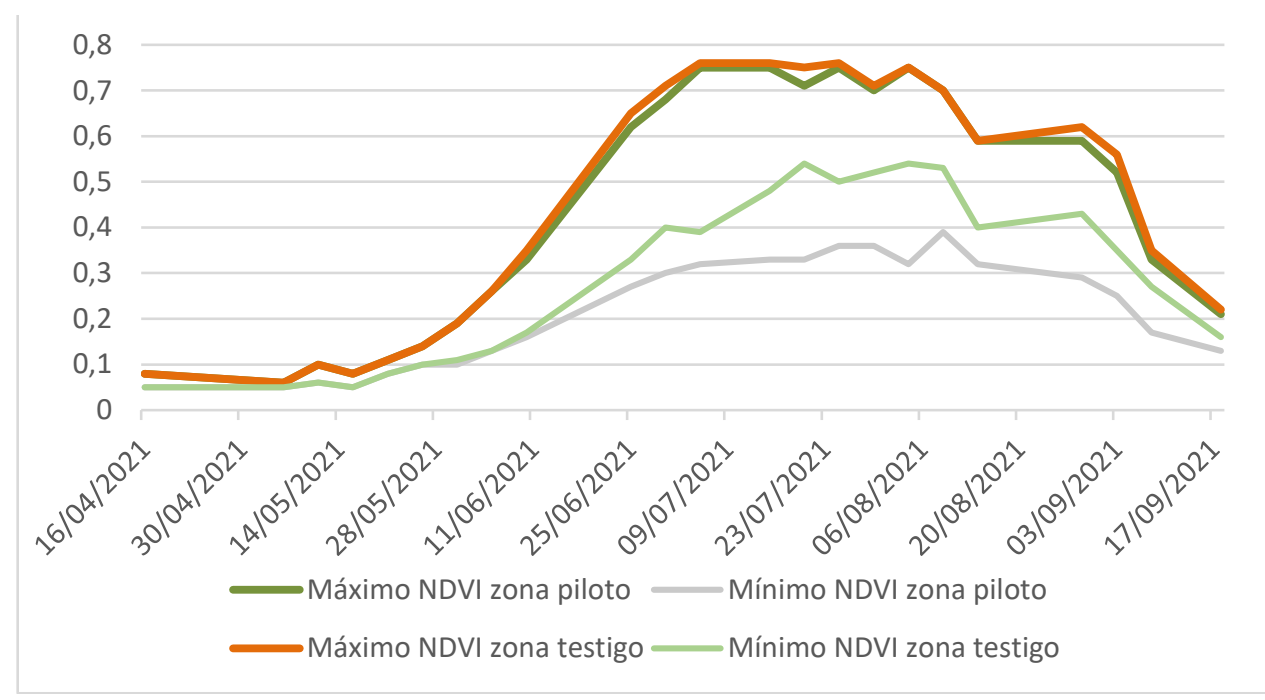

Figura 5. Gráfico de valores máximos y mínimos de NVDI en las dos subparcelas

En dicho gráfico aparecen dos irregularidades con respecto a la evolución del índice de vegetación NVDI de las parcelas. Los valores mínimos de la parcela piloto son muy bajos debido a la irregularidad del estado del suelo en esa parcela. Esta información se justifica en la siguiente figura.
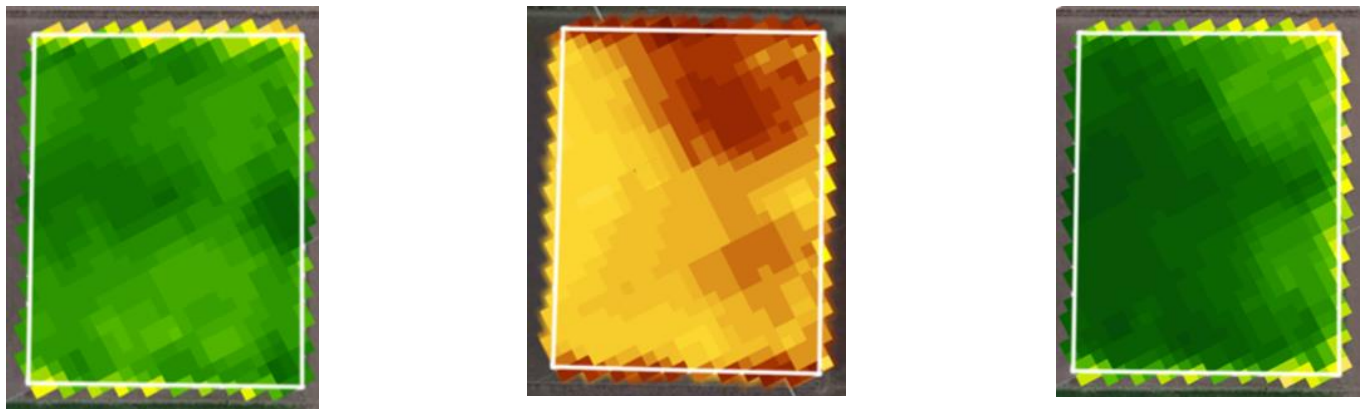

Índice vegetación NDVI de menor a mayor.

Fecha: 21/07/2018 Cultivo: Algodón Valores de NVDI máximo, medio y mínimo: $0.67,0,6$ y 0,4
Fecha: 05/06/2021 Cultivo: Algodón Imagen anterior al riego por pulsos dónde el primer riego fue el tradicional en toda la parcela
Fecha: 20/07/2021 Cultivo: Algodón. Valores de NVDI máximo, medio y mínimo: $0,71,0,65$ y 0,48

Figura 6. Imágenes de NVDI de circunstancias concretas sobre la parcela de ensayo. 


\section{Congreso Nacional de Riegos CARTAGENA 2021}

La irregularidad de la fertilidad del suelo es manifiesta desde una campaña anterior de algodón en el año 2018, tal como se aprecia en la imagen de la izquierda, donde todo fue regado con el mismo sistema de riego. Por tanto esa misma configuración se traslada a la campaña de 2021 como se puede verificar en la segunda imagen sobre el algodón de 2021 y antes de instalar la válvula de riego por pulsos, dónde el riego dado con el sistema tradicional en toda la parcerla manifiesta también dicha irregularidad. Por tanto, y a pesar de obtenerse una producción bruta en la zona testigo de $3.800 \mathrm{Kg} / \mathrm{ha}$ frente a una producción de la zona testigo ligeramente inferior $(3600 \mathrm{Kg} / \mathrm{ha})$, dichas producciones hubieran sido exactamente iguales con la fertilidad del suelo más homogénea. Esto fue comprobado con una corrección realizada a partir de muestras de pesadas parciales recogidas en puntos de similares valores de NVDI.

Por todo y adoptando en el cultivo del algodón un manejo más sostenible dónde se utilice el riego por pulsos y la fertirrigación en riego por surcos, se pueden conseguir buenas producciones con un ahorro mínimo de agua del $25 \%$ de agua del 34\% de nitrógeno. Sin embargo, se necesita continuar para conseguir más información que definan de manera más particularizada todos los parámetros del riego y así conseguir un techo de ahorro. En la bibliografía, y en casos experimentales parecidos se consigue un valor promedio del $35 \%$ de ahorro frente al manejo tradicional, con tasas de reducción de infiltración hasta de un $70 \%[6]$.

\section{Conclusiones}

Como resumen final se podría mencionar lo siguiente:

- El sistema de riego por surcos demuestra que puede ser un riego viable con posibilidades de mejora en la eficiencia de aplicación de riego.

- El sistema de pulsos de forma automatizada consigue considerables ahorros de agua en toda una campaña de riego de un cultivo herbáceo.

- El uso de la fertirrigación incorporada a la válvula automatizada de riego, permite una mayor eficiencia en la aplicación de abonos nitrogenados y facilita su aplicación sin generar costes añadidos.

- Es posible hacer más sostenible cualquier cultivo aplicando el este sistema automatizado no sólo desde el punto vista de rentabilidad sino desde el punto de vista ambiental.

- Un año de ensayo no es suficiente para llegar a un manejo óptimo del sistema. Por tanto, es necesario repetir en otras situaciones para conseguir mayores ahorros de agua y menores tasas de infiltración a las conseguidas inicialmente sobre el riego por surcos convencional.

\section{Referencias}

1. Ministerio de Agricultura, Pesca y Alimentación (MAPA). Informe sobre Regadíos en España. 2018. Gobierno de España

2. Walker, W.R. Guidelines for designing and evaluating surface irrigation systems. 1989. FAO Irrigation and Drainage Paper 45. Food and Agriculture Organitation of the United Nations. Rome, Italy.

3. C.W. Wood, L.J. Krutz, L. Falconer, H.C. Pringle III, B. Henry, T. Irby, J.M. Orlowski, C. J. Bryant, D.L. Boykin, R.L. Atwill, D.M. Pickelmann. Surge Irrigation Reduces Irrigation Requirements for Soybean on Smectitic Clay-Textured Soils. 2017. Crop Management. August 17.

4. Allen, Richard G., et al. Crop evapotranspiration-Guidelines for computing crop water requirements. 1998. FAO Irrigation and drainage paper 56. Food and Agriculture Organitation of the United Nations. Rome, Italy. Vol. 300, no 9.

5. Jana Harish, D.D. Pawar, K.D. Kale, R.R. Hasure and S.K. Dingre. Nutrient Availability in Bt. Cotton under Drip Fertigation. 2018. International Journal of Current Microbiology and Applied Sciences, April 7(04):3373-3379

6. Riza Kanber, Harun Köksal, Sermet Önder, Selim Kapur, Sebahattin Sahan. Comparison of surge and continuous furrow methods for cotton in the Harran plain. 2001. Agricultural Water Management 47, 119-135 